\title{
Analysis of Component Libraries for React JS
}

\author{
Mukthapuram Praneeth Reddy ${ }^{1}$, Sujata Priyambada Mishra ${ }^{2}$ \\ Student, Dept of Electronics and Communication, R.V. College of Engineering, Bangalore, INDIA -560102 ${ }^{1}$ \\ Professor, Dept of Electronics and Communication, R.V. College of Engineering, Bangalore, INDIA -560102²
}

\begin{abstract}
This purpose of this paper is to study and analyze different design frameworks for react js. Which include Material UI and Grommet and many more. This paper focuses on the importance of design frameworks in current web development world and the advantages of using each one as well as disadvantages. This is done with the help of react js along with Material UI and Grommet. There are many other frames works but these are the main stay in the evergrowing technology with a huge scope of improvement. This paper analyses the framework by looking into all the options provided to the user along with accessibility, re usability, community support and developer support. Material UI is generally used more than Grommet due to its high community support. Grommet is used mostly for company websites rather than for a small-scale projects.
\end{abstract}

KEYWORDS: Reactjs , Design framework , Material UI , Grommet , Ant Design

\section{INTRODUCTION:}

React Js is one of the most famous front end framework used by many of the developers.Since its launch by Facebook in 2013.In M-V-C (Model View Controller) design, it consolidates the view (V) layer. Facebook, Instagram, and a network of individual designers and associations support it.With increase in resource to use internet for people, there has been a need for online place for every business.There has also been increase in mobile usage which also led to mobile specific applications and websites.

For a website to be successful,It needs an User Interface. An Interface is a location on a website where a user interacts with it. The user interface (UI) is a critical component of creating an engaging website. A good User Interface Design combines visual design, interaction design, and information architecture in a cohesive manner.A developer must focus on design as much as other things.To meet the expectation of user,every business stated their own websites and mobile applications.This lead to a huge increase in creation of websites. With growing competition, the quality of a website should be improved. This is where

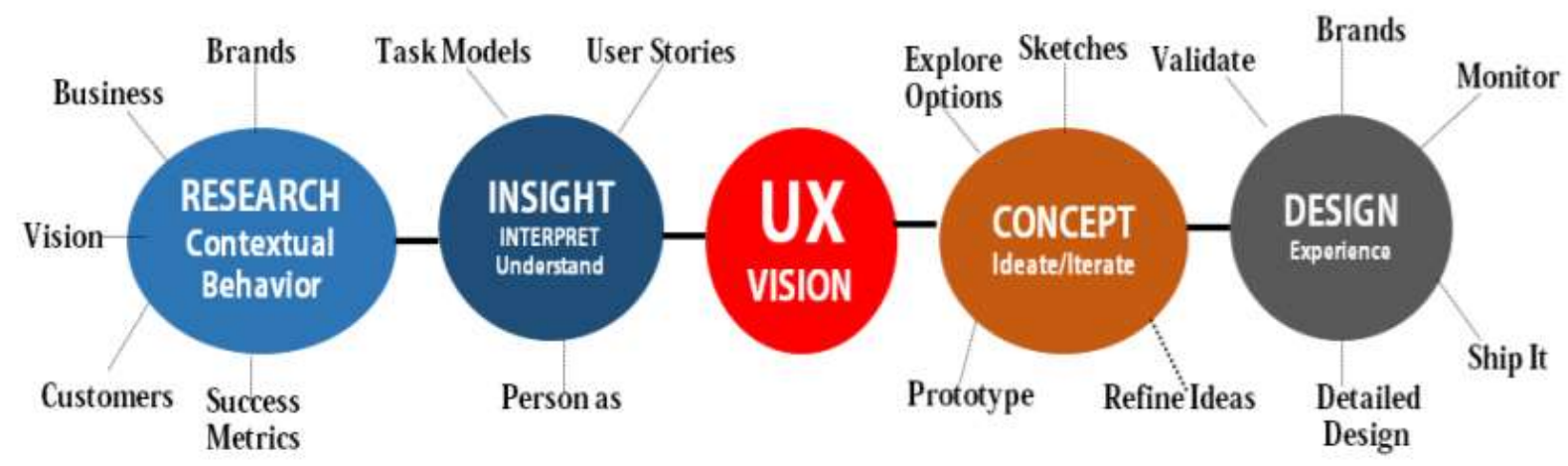

Figure 1. UI UX Design Interface Thinking

the component libraries come in.Each component library has their own unique style along with working technology.This gives the developer more options to meet the needs of their clients and attract more viewers.So the need arises to understand all the presently available component libraries.A component library is a collection of components that can be reused. It could be a folder within your application that contains common components that are utilized across the project. It might be a npm-based distributed package. It's also possible that it's part of a larger design scheme. 
Vol. 8, Issue 6, June 2021

DOI: $10.17148 /$ IARJSET.2021.8607

\section{Component Libraries:}

\section{Material UI:}

In 2017, Google released Material UI, which is based on Facebook's React framework and Google's Material Design standards. It's a time-consuming framework for building high-interactivity, mobile-first UIs with responsive designs for websites and apps. Material Design and Material UI are inextricably linked.Google uses Material Design in all of its products, while NASA, Unity, Amazon, JP Morgan, and others are using the newly popular Material UI, with the list growing in the future years.

Now looking into this library, it is very easy to customize leaving developers with less problems to worry in terms of design.Material UI gives three type of styling solution to choose from. One is Hook API and the others are styled components API and advanced API . All the types give same result, developers can choose one based on their usage.

Everything is documented on their official website and anyone can visit the website to check for any kind of information such as new additions, example codes. This is very important as it becomes easier to work with Material UI and learn it.It uses CSS as style language.CSS is one of the basic languages a developer learns to start with web development.As the use CSS is very high it becomes easier to style the components of Material UI.

The bundle size of an app refers to the amount of JavaScript that a user must download in order to use it. The larger the bundle, the longer it will take for a consumer to be able to see your application. If this becomes too slow, your users, particularly those with poorer internet connections, may become frustrated.This makes a importance parameter to consider when selecting library.MaterialUI has very less bundle size, less than $15 \mathrm{~Kb}$ gzipped.

All style are named with the Mui- prefix, so they shouldn't clash with other styles,If others are used.These are some of the features of Material UI.They are many more advantages and feature which can be considered.

\section{Grommet:}

Grommet is a React component library with mobile-first code components that are both responsive and accessible. It accomplishes this through its components, which serve as the library's building elements. Accessibility and responsiveness are incorporated into every key component.With its mobile-first, accessible, and responsive components, Grommet's major goal is to improve the developer experience and make it faster to build React applications. Grommet synchronizes a designer's and a developer's workflows to provide a seamless experience that anyone can use.

It also provides theme modes such as dark mode to gives user a more out of box experience.It can exist with other frame works with out interfering with global theme rather than work on selected components.It also features some layout components such as CSS flex-box and all its features as props.

It has a huge library of SVG icons which can used. Unlike many other libraries grommet has components for Data visualization such as tables,graphs,charts etc..It has an option to use code sandbox where developers can play around with several components to check for their functionality. Grommet is used by Netflix,IBM,HPE,Samsung and many others for their real life applications.It has a very big community support but still many of the developers are unaware of the library even though it has many advantages.

\section{Ant Design:}

Ant design is another react js UI framework backed by Alibaba corporation.It is know to be second most used UI framework for react.Similar to above all It has good community support and is well documented.Ant design specified their design value in the official documentation which helps the designer in opting for more user prone design and stress less about thinking.

They have a large component base with features ranging from normal buttons to data visualizations. This gives ant an edge over other frame works and libraries.It can support other front end frame works other than react like Angular and Vue. But there is a bit of issue with bundle size as it is on the heavier side which might effect the users with low internet speeds.It has support for multiple languages which will help developers from countries whose first language is not English.It also has powerful theme customization features. 
Vol. 8, Issue 6, June 2021

DOI: $10.17148 /$ IARJSET.2021.8607

Customizing the style and theming might be tough with the help of CSS but Styled components can be used to customize.For that the developer requires underlying Dom properties. This might be a hurdle for budding developers. The styles in ant design are scoped with ant prefix so they wont interfere with others but there are some global components that can be used to effect other styles.

\section{Results:}

Now all the libraries will be compared based on certain parameters to find their advantages and disadvantages.

\section{Popularity:}

This can be determined with the help of GitHub stars and forks.As there are more number of stars and forks tells how many people are actually using it.

\begin{tabular}{|c|c|c|}
\hline & & \\
\hline Material UI & $68.7 \mathrm{~K}$ & $224 \mathrm{~K}$ \\
\hline Grommet & $7.3 \mathrm{~K}$ & 888 \\
\hline Ant design & $72.2 \mathrm{~K}$ & $28.4 \mathrm{~K}$ \\
\hline
\end{tabular}

It can be concluded that Ant design has more number of Users and Material UI is nearly as popular as Ant-design whereas Grommet has very low number of users.

\section{Number of Contributors:}

Number of contributors in turn reveals the pace at which bugs can be resolved and the frame work can be developed.A technology must have good support if there is any chance to grow and sustain in the industry

Table2:Number of Contributors

\begin{tabular}{|c|c|}
\hline Framework & Contributors \\
\hline Material UI & 2193 \\
\hline Grommet & 281 \\
\hline Ant design & 1425 \\
\hline
\end{tabular}

It can be clearly deduced that Material UI has very good support for development followed by Ant design and then Grommet.

\section{Bundle Size:}

As mentioned already, Bundle size becomes important when user has low speed internet connection.So this parameter can be considered based on the target customers.If it is for enterprise based web pages Then It doesn't matter but if target users are mobile based then this must be a concern..

Table3:Bundle Size

\begin{tabular}{|c|c|}
\hline Framework & $\begin{array}{c}\text { Bundle } \\
\text { Size(mimified+gzipped) }\end{array}$ \\
\hline Material UI & $95 \mathrm{kB}$ \\
\hline Grommet & $560.9 \mathrm{kB}$ \\
\hline Ant design & $97.5 \mathrm{kB}$ \\
\hline
\end{tabular}

It can be conclude that ant design falls on a heavier side when it comes to bundle size when compared to other frame works

\section{Components:}

Number components directly influences the developers ability to be versatile and also look into future for improvements to the UI

Table4:Components

\begin{tabular}{|l|l|} 
Framework & Components \\
\hline
\end{tabular}


International Advanced Research Journal in Science, Engineering and Technology

Vol. 8, Issue 6, June 2021

DOI: $10.17148 / I A R J S E T .2021 .8607$

\begin{tabular}{|c|c|}
\hline Material UI & 35 \\
\hline Grommet & 45 \\
\hline Ant design & 60 \\
\hline
\end{tabular}

It can be seen that Ant design and Grommet have high number of components.

\section{Reusability:}

Code reusablility makes life easier for every developer.It also lessen the time need to work on the project.All the frameworks have good amount of support for reusable code.But ant design and Grommet have more reusable code resources when compared to Material UI.

\section{Support for Typescript:}

Typescript is language developed by Microsoft.It is a super set of Javascript and gives feature of static typing to it.It was stable released in 2021 which indicates it as fairly new language.All the frame works have typescript support which indicates that all the frameworks have good development and are on pace with current technologies.

\section{Cost:}

Cost effect the individual developers more than the enterprise.Even then it is better to use open source and free of cost technology than paying for a licence.All the frameworks are open source so it is free of cost where as Ant design has an option to pay for pro functionality which gives access to specific components that are not available in normal version.

\section{Conclusion and Future Scope:}

These are just some of the parameters that can be looked into when selecting a component library.There are many more parameter and factors which effect the decision.It is upto developer in the end to choose a library based on the need and scope for enhancement in the future.This paper just provides an introduction to different frameworks and what factors effect the decision making.Developer has to compromise with trade offs when an advantage of particular library has to be exploited.

As the online population increase there is a huge scope for this field.So it can be expected to develop leaps and bounds and many new libraries may arise giving developers more options to choose from.It is safe to say it can be more difficult job to choose a library rather than to develop with it.

\section{ACKNOWLEDGEMENT:}

Figure 1 is taken from reference [1].

\section{REFERENCES:}

[1] Heonsik Joo, "A Study on Understanding of UI and UX, and Understanding of Design According to User Interface Change" in.International Journal of Applied Engineering Research ISSN 0973-4562 Volume 12, Number 20 (2017) pp. 9931-9935.

[2] Darrel Greenhill, Jack Francik, Jay Kiruthika and Souheil Khaddaj, "UX Design in Web Applications" in , IEEE, (2016).

[3] Si-Jung Kim, Do-Eun Cho., Technology Trends for UX/UI of Smart Contents. The Korea Contents Association Review, 14(2016), 29-33.

[4] Won-Sung Sohn, Soon-Bum Lim, Sungho Woo., Curriculum development of UX based UI design for software, KOREA INFORMATION SCIENCE SOCIETY, 39(2012), 200-201.

[5] Jesse James Garrett's The Elements of User Experience: User-Centered Design for the Web and Beyond (2nd Edition).

[6] A Boduch's React Material-UI Cookbook.(1st Edition).

[7] ReactJS.org,' ReactJS official'. [Online]. Available: http://www.ReactJs.org.

[8] Material UI 'Material UI official' [Online]. Available: https://material-ui.com.

[9] Grommet 'Grommet Docs' [Online].Available: https://v2.grommet.io/.

[10] Ant-Design 'Ant-Design Docs’[Online].Available: https://ant.design/. 